Pacific Journal of Mathematics

THE CLASSIFICATION OF UNIFORM ALGEBRAS ON PLANE 


\title{
THE CLASSIFICATION OF UNIFORM ALGEBRAS ON PLANE DOMAINS
}

\author{
WILLIAM R. ZAME
}

Let $\Omega$ be an open subset of the complex plane. Denote by $\mathcal{O}(\Omega)$ the algebra of all holomorphic functions on $\Omega$, equipped with the topology of uniform convergence on compact set. The object of this paper is to provide a complete classification of all the closed subalgebras of $\mathcal{O}(\Omega)$ which contain the polynomials, and apply this classification to several concrete problems, including localness of these algebras, continuity of homomorphisms, and number of generators. It should be emphasized that no assumptions are made as to the connectivity of $\Omega$. In fact, in the cases of most interest, $\Omega$ will not be connected and some of the connected components of $\Omega$ will not be simply connected.

The most natural way in which such a classification might proceed would be to show, for such an algebra $A$, that the space $\Delta A$ of nonzero, continuous complex-valued homomorphisms of $A$, can be equipped with the structure of a one-dimensional complex-analytic space. Unfortunately, this is not generally the case. However, we can realize $\Delta A$ as the quotient of a certain Riemann surface, and equip it with a "pseudo-analytic structure", and this data serves to classify the algebra $A$. This is accomplished in $\S \S 1$ and 2 .

As with any classification scheme, it is natural to ask whether the scheme described here is a satisfactory one. This is partly a question of taste, but we suggest that a reasonable criterion is applicability to the solution of concrete problems. Following a suggestion of Kaplansky [11, p. 12] we consider three "test problem" which arise naturally in the study of uniform algebras:

(1) Is the algebra local on its homomorphism space?

(2) Is every complex-valued homomorphism of the algebra necessarily continuous?

(3) Is the algebra finitely-generated?

In $\S 3$, we use our classification scheme to show that the first two test problems always have affirmative solutions, and that the third test problem has an affirmative solution if $\Omega$ has only a finite number of connected components. We also give another application, suggested by work of Gamelin [8] and Bjork and de Paepe [7].

Some of our methods work in more general contexts, although the sharpest results do not obtain. In $\S 4$, we indicate the sort of result which can be obtained, and some limitations. 
Algebras of holomorphic functions on plane sets (or on Riemann surfaces) have been extensively studied by a number of authors, including Wermer $[17,18]$ Bishop [3, 5, 6], Royden [14], Bjork and de Paepe [7], Gamelin [9] and the author [19]. (This list is by no means complete.) The work described here is based on [19], which in turn is based on Bishop's development of Wermer's ideas on analytic structure.

Some of these results were announced in [20].

1. Stable algebras and homomorphism spaces. Throughout, we will let $\Omega$ denote a non-empty, open (not necessarily connected) subset of the complex plane $\boldsymbol{C}$. We denote by $\mathcal{O}(\Omega)$ the algebra of all holomorphic functions on $\Omega$. Equipped with the topology of uniform convergence on compact subsets of $\Omega, \mathcal{O}(\Omega)$ is a Frechet algebra; i.e., a complete, metrizable, locally convex, locally multiplicativelyconvex topological algebra. (For general information about such algebras, we refer to [15].) Evidently, any closed subalgebra of $\mathcal{O}(\Omega)$ is again a Frechet algebra.

If $A$ is a closed subalgebra of $\mathcal{O}(\Omega)$, we let $\Delta A$ denote the set of non-zero continuous, complex-valued homomorphisms of $A$. For $f \in A$, we let $\hat{f}: \Delta A \rightarrow C$ denote the Gelfand transform of $f$; i.e., the map given by $\hat{f}(\varphi)=\varphi(f)$ for each $\varphi \in \Delta A$. We give $\Delta A$ the finest topology which renders each of the maps $\hat{f}$ continuous. We let $\delta: \Omega \rightarrow \Delta A$ be the map which assigns to each point $x \in \Omega$ the homomorphism $\delta_{x}(f)=f(x)$. If $A$ contains the polynomials, then $\delta$ is a homeomorphism onto its range; we shall usually suppress $\delta$ and simply regard $\Omega$ as a subset of $\Delta A$.

Our classification of subalgebras of $\delta(\Omega)$ will utilize the structure theory of certain simple subalgebras of $\mathscr{O}(\Omega)$ and we begin by describing these. We say a closed subalgebra $B$ of $\mathscr{O}(\Omega)$ is stable if it contains the polynomials and is closed under differentiation with respect to the coordinate function $z$. Stable algebras may be constructed in the following way. Let $\Omega=\mathbf{U} \Omega_{\alpha}$ be a partition of $\Omega$ into (disjoint) open and closed subsets (so that each $\Omega_{\alpha}$ is a union of connected components of $\Omega$ ). For each $\alpha$, let $\Omega_{\alpha}^{\prime}$ be a connected open subset of $C$ which is the union of $\Omega_{\alpha}$ with some of the bounded, connected components of $C \mid \Omega_{\alpha}$. (Note that the sets $\Omega_{\alpha}^{\prime}$ need not be disjoint.) Let $B$ be the set of holomorphic functions $f$ on $\Omega$ such that $f \backslash \Omega_{\alpha}$ belongs to $\mathscr{O}\left(\Omega_{\alpha}^{\prime}\right) \mid \Omega_{\alpha}$ for each $\alpha$. It is evident that $B$ is a stable subalgebra of $\mathcal{O}(\Omega)$, and that $\Delta B$ is the disjoint union of the sets $\Omega_{\alpha}^{\prime}$, so $\Delta B$ naturally carries of a (not necessarily connected) Riemann surface. The following result shows that all the stable subalgebras of $\mathcal{O}(\Omega)$ arise in this way. Virtually the same result was given in [19], although formulated for compact sets rather than 
open sets, so we shall omit the proof.

THeOREM 1. Let $B$ be a stable subalgebra of $\mathcal{O}^{\prime}(\Omega)$ and let $\Delta B=$ $\cup Y_{\alpha}$ be the decomposition of $\Delta B$ into connected components. Then:

(i) for each $\alpha, \widehat{z} \mid Y_{\alpha}$ is a homeomorphism onto a connected open subset of $\boldsymbol{C}$;

(ii) for each $\alpha, \Omega_{\alpha}=\widehat{z}\left(Y_{\alpha} \cap \Omega\right)$ is an open and closed subset of $\Omega$ and $\Omega_{\alpha}^{\prime}=\widehat{z}\left(Y_{\alpha}\right)$ is the union of $\Omega_{\alpha}$ with some of the bounded, connected components of $C \backslash \Omega_{\alpha}$;

(iii) $B=\left\{f \in \mathcal{O}(\Omega): f\left|\Omega_{\alpha} \in \mathcal{O}\left(\Omega_{\alpha}^{\prime}\right)\right| \Omega_{\alpha}\right.$ for each $\left.\alpha\right\}$.

Now let $A$ be a closed subalgebra of $\mathscr{O}(\Omega)$ which contains the polynomials and let $B$ be the smallest stable subalgebra of $\mathscr{O}(\Omega)$ which contains $A$. It is evident that $B$ is the closure of the algebra generated by the elements of $A$ and all their derivatives (of all orders). Let $\rho: \Delta B \rightarrow \Delta A$ be the restriction map. It will be convenient to suppress the Gelfand transform for functions acting on $\Delta B$ and retain it for functions acting on $\triangle A$. Thus we regard an element $f \in A$ as a function on $\Delta B$, while its Gelfand transform $\hat{f}$ is a function on $\Delta A$, and $\hat{f} \circ \rho=f$.

For each compact subset $K \subset \Delta B$ we define a semi-norm $\|\cdot\|_{K}$ on $B$ by:

$$
\|f\|_{K}=\sup \{|f(x)|: x \in K\} .
$$

The $B$-convex hull of the compact set $K$ is

$$
\widetilde{K}_{B}=\left\{x \in \Delta B:|f(x)| \leqq\|f\|_{K} \text { for each } f \in B\right\} .
$$

We say that an arbitrary subset $E \subset \Delta B$ is $B$-convex if $\widetilde{K}_{B}$ is a subset of $E$ whenever $K$ is a compact subset of $E$. (We make the obvious, similar definitions for any algebra of functions on any space.) Note that an open subset $W$ of $\Delta B$ is $B$-convex exactly when $\Delta B \backslash W$ has no compact, connected components.

The following result expresses the relationship between $\Delta B$ and $\Delta A$. Again, virtually the same result was given in [19], so we omit the proof.

THEOREM 2. Let $A, B$ and $\rho$ be as above.

(i) If $V$ is an open, relatively compact, B-convex subset of $\Delta B$ then $\rho(V)$ is a relatively compact, A-convex subset of $\triangle A$;

(ii) the mapping $\rho: \Delta B \rightarrow \Delta A$ is continuous and onto;

(iii) if $x, y$ are in $\Delta B$ and $\rho(x)=\rho(y)$ then $z(x)=z(y)$;

(iv) the set $\mathscr{E}_{0}=\{(x, y) \in \Delta B \times \Delta B: x \neq y$ and $\rho(x)=\rho(y)\}$ is $a$ countable, discrete subset of $\Delta B \times \Delta B$;

(v) if $\rho$ is one-to-one, then $A=B$. 
A number of remarks are in order at this point. Note first that it is not asserted that $\rho: \Delta B \rightarrow \Delta A$ is a quotient map; i.e., that the topology on $\triangle A$ is the finest which renders $\rho$ continuous. (In fact, we do not know whether or not this is the case, although if $W$ is an open, relatively compact subset of $\Delta B$ then it is easy to see that $\rho: W \rightarrow \rho(W)$ is a quotient map.) This will cause some inconvenience, but is not really a serious problem. Second, although $\mathscr{E}_{0}$ is a discrete subset of $\Delta B \times \Delta B$, the sets $\Sigma=\left\{y \in \Delta A: \rho^{-1}(y)\right.$ is not a singleton $\}$ and $\rho^{-1}(\Sigma)=\left\{x \in \Delta B: \rho^{-1} \rho(x) \neq\{x\}\right\}$ need not be discrete. This can be seen from the following example, which illustrates the sort of pathology which may be present.

Let $D_{k}$ denote the open disk with center 0 and radius $k$; let $\bar{D}_{k}$ be its closure. Set $\Omega=D_{1} \cup\left(D_{2} \mid \bar{D}_{1}\right)$ and set

$$
B=\left\{f \in \mathcal{O}(\Omega): f\left|\left(D_{2} \backslash \bar{D}_{1}\right) \in \mathcal{O}\left(D_{2}\right)\right|\left(D_{2} \mid \bar{D}_{1}\right)\right\} .
$$

Thus for each $f \in B$ there is a unique $\hat{f} \in \mathscr{O}\left(D_{2}\right)$ such that $f \mid\left(D_{2} \mid \bar{D}_{1}\right)=$ $\hat{f} \mid\left(D_{2} \backslash \vec{D}_{1}\right)$. It is evident that $B$ is a stable subalgebra of $\mathcal{O}(\Omega)$ and that $\Delta B$ may be identified with the disjoint union of $D_{1}$ and $D_{2}$. Choose an infinite discrete subset $\left\{x_{n}\right\} \subset D_{1}$ and set $A=\left\{f \in B: f\left(x_{n}\right)=\right.$ $\hat{f}\left(x_{n}\right)$ for each $\left.n\right\}$. It is easy to see that $\Delta A$ is the space formed from the disjoint union of $D_{1}$ and $D_{2}$ by identifying each of the points $x_{n} \in D_{1}$ with the corresponding point $x_{n} \in D_{2}$. Notice that neither $\Sigma$ nor $\rho^{-1}(\Sigma)$ are discrete sets. (In fact, by using a similar construction with an infinite family of disks, it is possible to construct an example in which both $\Sigma$ and $\rho^{-1}(\Sigma)$ are dense.) It is evident that $\Delta A$ cannot be equipped with the structure of a complex-analytic space, since the set of singularities would not be discrete. However, the image in $\Delta A$ of each relatively compact, open subset of $\Delta B$ does admit the structure of a complex-analytic space; this observation is the key to our classification scheme.

Finally, we note that Theorems 1 and 2, taken together, provide us with a method of computing the stable algebra $B$ directly from $A$. This may be seen most easily by considering a special case. As above, let $D_{k}$ be the open disk with center 0 , radius $k$, let $\bar{D}_{k}$ be its closure, and set $\Omega=D_{1} \cup\left(D_{2} \mid \bar{D}_{1}\right)$. It is easily seen that there are precisely three stable subalgebras of $\mathcal{O}(\Omega)$ :

$$
\begin{aligned}
& B_{1}=\mathcal{O}\left(D_{2}\right) \mid \Omega, \\
& B_{2}=\left\{f \in \mathcal{O}(\Omega): f\left|\left(D_{2} \mid \bar{D}_{1}\right) \in \mathcal{O}\left(D_{2}\right)\right|\left(\bar{D}_{2} \backslash D_{1}\right)\right\}, \\
& B_{3}=\mathcal{O}(\Omega) .
\end{aligned}
$$

Given a closed subalgebra $A$ of $\mathcal{O}(\Omega)$ which contains the polynomials, it is a simple matter to determine $B$, the smallset stable algebra containing $A$. For it follows from Theorem 2 that the functions in 
$B$ must have precisely the same extension properties as functions in $A$, so there are precisely three situations which can arise:

(1) every function in $A$ extends to $D_{2}$, in which case $B=B_{1}$;

(2) for every function $f$ in $A, f \mid\left(D_{2} \mid \bar{D}_{1}\right)$ extends to a function $\hat{f} \in \mathcal{O}\left(D_{2}\right)$, and there is function $g \in A$ such that $\hat{g}$ does not agree with $g$ on $D_{1}$; in this case $B=B_{2}$;

(3) there is a function $f \in A$ for which $f \mid\left(D_{2} \mid \bar{D}_{1}\right)$ does not extend to a function in $\mathcal{O}\left(D_{2}\right)$, in which case $B=B_{3}$.

The same sort of analysis may be carried out in the general case, although we shall not do so. The point is simply that the algebra $B$ can actually be recovered simply from $A$ by extension properties and is not an additional construct. (Note: the possibility of recovering $B$ from extension properties of functions in $A$ will also play an important role in the proofs of Theorems 3 and 7.)

2. Pseudo-analytic structures and the classification theorem. As was mentioned in the introduction, the most natural way to classify the closed subalgebras $A$ of $\mathscr{O}(\Omega)$ which contain the polynomials would be to endow $\triangle A$ with the structure of a one-dimensional complex-analytic space. As we show in $\S 1$, this is not always possible because $\Delta A$ may have too many singularities. It turns out however, that if we restrict attention to a relatively compact open subset $W$ of $\Delta B$, then the image $\rho(W)$ under the natural restriction $\rho: \Delta B \rightarrow \Delta A$ can indeed be endowed with the structure of a onedimensional complex-analytic space. If we cover $\Delta B$ with such open sets, we obtain a collection of "local" analytic space structures on $\triangle A$; of course these structures are "local" relative to $\Delta B$, not relative to $\Delta A$ (since $\rho$ is not an open mapping). Patching these structures together produces what we will call a pseudo-analytic structure. There is one slight bit of inconvenience, since we do not know whether or not $\Delta A$ carries the quotient topology. It turns out to be simpler to deal with the quotient topology; recall, however, that for a relatively compact open subset $W$ of $\Delta B$, the quotient topology on $\rho(W)$ does agree with the induced topology from $\Delta A$.

It will be convenient to formulate the general notion of a pseudo-analytic structure so as to be applicable to algebras on Riemann surfaces. To this end, let $R$ be a (not necessarily connected) Riemann surface and let $\mathscr{E}$ be an equivalence relation on $R$, thought of as a subset of $R \times R$. We say that $\mathscr{E}$ is admissible if $\mathscr{E}$ if a closed subset of $R \times R$ and the intersection of $\mathscr{E}$ with the complement of the diagonal is a countable discrete subset of $R \times R$. If $\mathscr{E}$ is an admissible equivalence relation on $R$, let $R / \mathscr{E}$ denote the quotient space and $\pi: R \rightarrow R / \mathscr{E}$ the quotient map. We give $R / \mathscr{C}$ the quotient topology; i.e., the finest topology which renders the map $\pi$ continuous. (This 
topology is Hausdorff, but need not be metrizable or locally compact.) By a pseudo-analytic structure on $R / \mathscr{E}$, we mean a subset $\mathscr{S}$ of $\mathscr{C}_{R / \mathscr{E}}$, the sheaf of continuous functions on $R / \mathscr{E}$, such that $(\pi(W)$, $\mathscr{S} \mid \pi(W))$ is a one-dimensional complex-analytic space and $\pi: W \rightarrow$ $\pi(W)$ is holomorphic for each open, relatively compact subset $W$ of $R$. (Here, we regard $\mathscr{S} \mid \pi(W)$ as a subset of $\mathscr{C}_{\pi(W)}$; i.e., the elements of $\mathscr{S} \mid \pi(W)$ are to be thought of as germs of continuous functions on $\pi(W)$.) We emphasize that it is not assumed that $\mathscr{S}$ is a subsheaf of $\mathscr{C}_{R / \mathscr{E}}$; in fact, $\mathscr{S}$ will not generally be an open subset of $\mathscr{C}_{R / \mathscr{E}}$. We denote by $\Gamma\left(R / \mathscr{E}, \mathscr{C}_{R / \mathscr{E}}\right)$ the space of sections of $\mathscr{C}_{R / \mathscr{S}}$ (which we usually regard simply as continuous functions), and by $\Gamma(R / \mathscr{E}, \mathscr{S})$ the subspace of sections which lie in $\mathscr{S}$ at each point. Notice that $\{f \circ \pi: f \in \Gamma(R / \mathscr{E}, \mathscr{S})\}$ is a subset of the algebra $\mathcal{O}(R)$ of all holomorphic functions on $R$.

In order to treat algebras on open subsets $\Omega$ of the complex plane, we introduce one further notion. By a regular triple for $\Omega$ we mean a triple $(R, \mathscr{E}, \mathscr{S})$ where $R=\Delta B$ is the homomorphism space of a stable subalgebra of $\mathscr{O}(\Omega)$ (we regard $\Omega$ as an open subset of $R), \mathscr{E}$ is an admissible equivalence relation on $R=\Delta B$ such that $z(x)=z(y)$ whenever $(x, y) \in \mathscr{E}$, and $\mathscr{S}$ is a pseudo-analytic structure on $R / \mathscr{E}$ such that $z \circ \pi^{-1}$ is holomorphic on $\pi(W)$ for each open, relatively compact subset $W$ of $\Delta B$. (Note that $z \circ \pi^{-1}$ is well-defined because of our restriction on $\mathscr{E}$.) We can now give our Classification Theorem for closed subalgebras of $\mathscr{O}(\Omega)$ which contain the polynomials.

THEOREM 3. Let $\Omega$ be an open subset of the complex plane. The $\operatorname{map}$

$$
\gamma:(R, \mathscr{E}, \mathscr{S}) \longmapsto\{f \circ \pi: f \in \Gamma(R / \mathscr{E}, \mathscr{S})\} \mid \Omega
$$

is a bijection between the regular triples for $\Omega$ and the closed subalgebras of $\mathcal{O}(\Omega)$ which contain the polynomials.

Proof. It is convenient to begin by constructing the inverse of $\gamma$, which is a map from algebras to triples. To this end, let $A$ be a closed subalgebra of $\mathcal{O}(\Omega)$ which contains the polynomials. Let $B$ be the smallest stable subalgebra of $\mathscr{O}(\Omega)$ which contains $A$ and set $R=\Delta B, \mathscr{E}=\{(x, y) \in R \times R: f(x)=f(y)$ for all $f \in A\}$. In view of Theorems 1 and $2, R$ is a Riemann surface, $\mathscr{E}$ is an admissible equivalence relation on $R$ and $z(x)=z(y)$ for $(x, y)$ in $\mathscr{E}$. Note that $R / \mathscr{E}$ and $\Delta A$ agree as sets, although the topology of $R / \mathscr{E}$ may be finer than the topology of $\Delta A$. For each $f \in A$, we may regard the Gelfand transform $\hat{f}$ as a continuous function on $R / \mathscr{E}$. Let $\pi: R \rightarrow R / \mathscr{E}$ be the quotient map.

We now wish to construct a pseudo-analytic structure on $R / \mathscr{E}$. 
We first use [10, VII, C7] to choose and fix functions $f_{1}, f_{2}$ in $A$ such that $\varphi=\left(\widehat{z}, \hat{f}_{1}, \hat{f}_{2}\right): R / \mathscr{E} \rightarrow C^{3}$ is a one-to-one map. Now let $W$ be an open, relatively compact, $B$-convex subset of $R=\Delta B$. We are going to construct an analytic structure on $\pi(W)$ by imbedding it in $C^{N}$ for sufficiently large $N$.

Since $W$ is relatively compact, $\pi$ and $\varphi$ identify only a finite number of pairs of points in $W$. Hence $\pi(W)$ is locally compact and $\varphi \mid \pi(W)$ is a homeomorphism onto the locally compact subset $\varphi(\pi(W))$ of $\boldsymbol{C}^{3}$. We can therefore find an open subset $Q$ of $\boldsymbol{C}^{3}$ such that $\varphi(\pi(W))$ is a closed subset of $Q$. Thus, $\varphi \circ \pi$ is a proper mapping of $W$ into $Q$, so $Y=\varphi \circ \pi(W)$ is a closed subvariety of $Q$. Since the first component of $\varphi \circ \pi$ is $z$, it follows that $\varphi \circ \pi$ maps the connected components of $W$ in a one-to-one biholomorphic fashion onto the irreducible branches of $Y$, which are therefore non-singular.

Now, every $g \in A$ defines a continuous function $\widetilde{g}=g \circ(\varphi \circ \pi)^{-1}=$ $\hat{g} \circ \varphi^{-1}$ on $Y$, which is holomorphic at the regular points and thus in particular is weakly holomorphic on $Y$. Let $\widetilde{A}=\{\widetilde{g}: g \in A\}$. Since $Y$ has only a finite number of singularities, the space $\mathscr{O}(Y)$ of holomorphic functions on $Y$ is of finite co-dimension in the space $\mathscr{O}^{w}(Y)$ of weakly holomorphic functions on $Y$ [13]. Hence $\widetilde{A} \cap \mathcal{O}(Y)$ is also of finite co-dimension in $\widetilde{A} \cap \mathcal{O}^{w}(Y)=\widetilde{A}$. Let $f_{3}, \cdots, f_{r}$ be elements of $A$ such that $\hat{f}_{3}, \cdots, \hat{f}_{r}$ and $\widetilde{A} \cap \mathcal{O}(Y)$ span $\widetilde{A}$. Set $\Phi=\left(\hat{z}, \hat{f}_{1}, \hat{f}_{2}, \cdots, \hat{f}_{r}\right)$ : $\pi(W) \rightarrow C^{r+1}$. As above, we see that $\Phi$ is a homeomorphism of $\pi(W)$ onto a closed subvariety $V=\Phi(\pi(W))$ of some open set $U \subseteq C^{r+1}$. Let $\mathscr{O}_{V}$ be the structure sheaf of $V$ and set $\mathscr{S}_{\pi(W)}=\Phi^{-1}\left(\mathscr{O}_{V}\right)$, so that $\left(\pi(W), \mathscr{S}_{\pi(W)}\right)$ is a one-dimensional complex-analytic space. Clearly, $\pi: W \rightarrow \pi(W)$ is holomorphic, and $z \circ \pi^{-1}: \pi(W) \rightarrow C$ is also holomorphic.

Our construction guarantees that the elements of $\hat{A}$ are holomorphic on $\pi(W)$, separate points and determine the analytic structure. Moreover, since Theorem 2 guarantees that $\pi(W)$ is $\hat{A}$-convex (recall that the topologies $\Delta A$ and $R / \mathscr{E}$ agree on $\pi(W)$ ), the Oka-Weil theorem allows us to conclude that $\hat{A} \mid \pi(W)$ is dense in the algebra $\Gamma\left(\pi(W), \mathscr{S}_{\pi(W)}\right)$ of all holomorphic functions on $\pi(W)$.

Notice that if $W^{\prime}$ is another open, relatively compact, $B$-convex subset of $\Delta B$, then the sheaves $\mathscr{S}_{\pi(W)}$ and $\mathscr{S}_{\pi\left(W^{\prime}\right)}$ agree on $\pi(W) \cap \pi\left(W^{\prime}\right)$, since they are each determined by elements of $\hat{A}$.

We can now define the desired subset $\mathscr{S}$ of $\mathscr{C}_{R / \mathscr{b}}$. For each point $x$ in $R / \mathscr{E}$, we let ${ }_{x} \mathscr{S}$ be the set of all elements $\alpha$ in the stalk ${ }_{x} \mathscr{C}_{R / \mathscr{B}}$ such that $\alpha \mid \pi(W) \in{ }_{x} \mathscr{S}_{\pi(W)}$ for each open, relatively compact, $B$-convex subset $W$ of $\Delta B$. We then set $\mathscr{S}=\mathrm{U}_{x} \mathscr{S}$. Our previous remaks guarantee that $\mathscr{S} \mid \pi(W)=\mathscr{S}_{\pi(W)}$ for each open, relatively compact, $B$-convex subset $W$ of $\Delta B$. This guarantees that $\mathscr{S}$ is a pseudo-analytic structure on $R / \mathscr{E}$; by our previous remarks, $(R, \mathscr{E}, \mathscr{S})$ is in fact a regular triple for $\Omega$. 
We now assert that, regarding $A$ as an algebra of functions on $R=\Delta B$, we have $A=\{f \circ \pi: f \in \Gamma(R / \mathscr{E}, \mathscr{S})\}$. The containment $A \subset$ $\{f \circ \pi\}$ follows directly from our construction. On the other hand, if $f \in \Gamma(R / \mathscr{E}, \mathscr{S})$ and $K$ is an arbitrary compact subset of $\Delta B$, we can choose an open, relatively compact subset $W$ of $\Delta B$ which contains $K$. Since $\hat{A} \mid \pi(W)$ is dense in $\Gamma(\pi(W), \mathscr{S} \mid \pi(W))$, we can choose a sequence $\left\{g_{n}\right\}$ of elements of $A$ such that $\hat{g}_{n} \mid \pi(W)$ converges to $f \mid \pi(W)$ uniformly on compact sets. In particular, $g_{n} \mid K$ converges uniformly to $f \circ \pi \mid K$. Since $K$ is arbitrary and $A$ is closed, we conclude that $f \circ \pi \in A$, which yields the desired equality. If we now restrict to $\Omega$, we see that we have proved: for each closed subalgebra $A$ of $\mathscr{O}(\Omega)$ which contains the polynomials, there is a triple $(R, \mathscr{E}, \mathscr{S})$ which is regular for $\Omega$ such that $\gamma(R, \mathscr{E}, \mathscr{S})=A$. That is, the map from algebras to triples is a right inverse to $\gamma$; we need to show that it is also a left inverse.

To this end, let $(R, \mathscr{F}, \mathscr{T})$ be a regular triple for $\Omega$, where $R=\Delta B$ for $B$ a stable subalgebra of $O(\Omega)$. Set $A=\{f \circ \pi: f \in$ $\Gamma(R(\mathscr{F}, \mathscr{T})\}$. It follows from Theorem 1 that every connected component of $R \backslash \Omega$ is compact, so the restriction $\mathscr{O}(R) \rightarrow \mathscr{O}(\Omega)$ is a homeomorphism onto its range. We can thus identify $A$ with the subset $A \mid \Omega=\gamma(R, \mathscr{F}, \mathscr{T})$ of $\mathscr{O}(\Omega)$, and we need to establish five things:

(i) $A$ is a closed subalgebra of $B=\mathscr{O}(R)$;

(ii) the function $z$ belongs to $A$;

(iii) $B$ is the smallest stable algebra containing $A$;

(iv) $\mathscr{F}$ is the equivalence relation associated to $A$;

(v) $\mathscr{T}$ is the pseudo-analytic structure associated to $A$.

That $A$ is a subset of $B=\mathscr{O}(R)$ was already observed in the definition of pseudo-analytic structure; it is in fact a subalgebra since the sheaves $\mathscr{T} \mid \pi(W)$ are all sheaves of algebras. To see that $A$ is closed, let $\left\{f_{n}\right\}$ be a sequence of functions in $\Gamma(R / \mathscr{F}, \mathscr{T})$ and suppose that $\left\{f_{n} \circ \pi\right\}$ converges to a function $G$. Since $G$ respects the equivalence relation $\mathscr{F}$ and $R / \mathscr{F}$ has the quotient topology, there is a continuous function $g$ on $R / \mathscr{F}$ such that $G=g \circ \pi$. Moreover, for each open, relatively compact subset $W$ of $\Delta B$, the sequence $f_{n} \mid \pi(W)$ converges to $g \mid \pi(W)$, uniformly on compact sets, so $g$ is analytic on $\pi(W)$. Hence $g \in \Gamma(R / \mathscr{F}, \mathscr{T})$, so $G=g \circ \pi$ belongs to $A$, as desired. This proves (i).

To see that $z \in A$, note that for each open, relatively compact subset $W$ of $\Delta B, z \circ \pi^{-1}$ is holomorphic on $\pi(W)$. Hence $z \circ \pi^{-1} \epsilon$ $\Gamma(R / \mathscr{E}, \mathscr{F})$ so $z=z \circ \pi^{-1} \circ \pi \in A$.

To establish (iii), (iv) and (v), we first prove the following claim: If $W$ is an open, relatively compact, $B$-convex subset of $\Delta B$, then $\Gamma(R / \mathscr{F}, \mathscr{T})$ is dense in $\Gamma(\pi(W), \mathscr{T} \mid \pi(W))$. To see this, let $W_{1}$ be 
another relatively compact, open, $B$-convex subset of $\Delta B$ with $W_{1} \supseteq \bar{W}$. Since $\Delta B \backslash W$ has no compact components, it follows that $\pi\left(W_{1}\right) \backslash \pi(W)$ also has no compact components. Moreover, every. irreducible branch of $\pi(W)$ is an open subset of an irreducible branch of $\pi\left(W_{1}\right)$. Thus, $\Gamma\left(\pi\left(W_{1}\right), \mathscr{T} \mid\left(\pi\left(W_{1}\right)\right)\right.$ is dense in $\Gamma(\pi(W), \mathscr{T} \mid(\pi(W))$. It now follows by the usual Runge approximation argument that $\Gamma(R / \mathscr{F}, \mathscr{T})$ is dense in $\Gamma(\pi(W), \mathscr{T} \mid(\pi(W))$, as claimed.

To establish (iii), let $\alpha: A \rightarrow C$ be a continuous homomorphism. Then there is a compact subset $K$ of $\Delta B$ such that $|\alpha(f)| \leqq\|f\|_{K}$ for each $f$ in $A$. Choose a relatively compact, open $B$-convex set $W \supset K$. Then $\alpha$ induces a homomorphism $\Gamma(R / \mathscr{F}, \mathscr{T}) \rightarrow C$; since $\Gamma(R / \mathscr{F}, \mathscr{T})$ is dense in $\Gamma(\pi(W), \mathscr{T} \mid(\pi(W))$, we obtain a continuous homomorphism $\Gamma(\pi(W), \mathscr{T} \mid(\pi(W)) \rightarrow \boldsymbol{C}$, which is necessarily given by evaluation at a point of $\pi(W)$. Hence $\alpha$ is given by evaluation at a point of $W$ (which of course may not be unique). That is to say, the natural map $\rho: \Delta B \rightarrow \Delta A$ is onto. Since it is clear from the above claim that $\rho(x)=\rho(y)$ if and only if $(x, y) \in \mathscr{F}$, and $\mathscr{F}$ is an admissible equivalence relation, we may use Theorem 2 to conclude that $B$ is the smallest stable algebra containing $A$, which is assertion (iii). The above remark guarantees that $\mathscr{F}$ is the equivalence relation associated to $A$, which is assertion (iv). Finally, since $\Gamma(R / \mathscr{F}, \mathscr{T})$ is dense in $\Gamma(\pi(W), \mathscr{T} \mid(\pi(W))$ for each open, relatively compact, $B$-convex subset $W$ of $\Delta B$, we see that $\mathscr{T}$ is the pseudoanalytic structure associated to $A$, which is assertion (v).

We conclude that the map from algebras to triples is indeed a two-sided inverse to $\gamma$, which completes the proof.

In general, a pseudo-analytic structure may be quite complicated. However, there is one very important situation in which pseudoanalytic structures are easy to describe. Let $B$ be a stable subalgebra of $\mathscr{O}(\Omega)$ and let $\mathscr{E}$ be an admissible relation on $\Delta B$ such that $z(x)=$ $z(y)$ for all $(x, y)$ in $\mathscr{E}$. Assume that the quotient mapping $\pi: \Delta B \rightarrow$ $\Delta B / \mathscr{E}$ has finite fibers. (Note that this will be the case whenever $\Omega$, and hence $\Delta B$, has only finitely many connected components.) Let $\Sigma=\left\{y \in \Delta B / \mathscr{E}: \pi^{-1}(y)\right.$ is not a singleton $\}$. For each $y \in \Sigma$, write $\pi^{-1}(y)=\left\{x_{1}, \cdots, x_{n}\right\}$ where $n \geqq 2$ (and $n$ may depend on $y$ ). Since $\mathscr{E}$ is an admissible relation, we may choose connected open sets $W_{1}, \cdots, W_{n}$ in $\Delta B$ such that $x_{i} \in W_{i}$ for each $i$, and $\pi$ is one-to-one on $\mathrm{U}\left(W_{i} \backslash\left\{x_{i}\right\}\right)$. Now choose a local subvariety $V_{y}$ of $\boldsymbol{C}^{m}$ (for some $m$ depending on $y$ ) with $n$ irreducible branches $V_{y}^{1}, \cdots, V_{y}^{n}$ each of which is one-dimensional and non-singular, and which meet only at the origin. After shrinking the $W_{i}$ and $V_{y}^{i}$ if necessary, we can choose a holomorphic map $g: \cup W_{i} \rightarrow V_{y}$ such that $g$ maps $W_{i}$ biholomorphically onto $V_{y}^{i}$ and $g\left(x_{i}\right)=0$ for each $i$. 
We can define a pseudo-analytic structure $\mathscr{S}$ on $\Delta B / \mathscr{E}$ by setting, for $y \in \Sigma,{ }_{y} \mathscr{S}=\left(g \circ \pi^{-1}\right)^{-1}\left(\mathscr{O}_{V_{y}}\right)$ where $\mathscr{O}_{V_{y}}$ is the sheaf of holomorphic functions on $V_{y}$. (This makes sense since $g \circ \pi^{-1}$ is a continuous map from $\pi\left(\mathbf{U} W_{i}\right)$ to $V_{y}$ which maps $y$ to 0 .) For $y \notin \Sigma$, we set ${ }_{y} \mathscr{S}=\pi\left(_{\pi^{-1}(y)} \mathscr{O}_{\Delta B}\right)$, where $\mathscr{O}_{\Delta B}$ is the sheaf of holomorphic functions on $\triangle B$. It is easily checked that $\mathscr{S}=\bigcup_{y} \mathscr{S}$ is a pseudo-analytic structure on $\Delta B / \mathscr{E}$; in fact $(\Delta B, \mathscr{E}, \mathscr{S})$ is a regular triple for $\Omega$, and all regular triples arise in this way. Notice that this construction is merely a more sophisticated version of the example described in $\S 1$; we have simply specified, at each "singular point" of $\Delta B / \mathscr{E}$, the germ of a one-dimensional analytic space, and decreed that all other points of $\Delta B / \mathscr{E}$ be "regular points".

3. Applications. In order to demonstrate the usefulness of our classification scheme, we give a number of applications. Throughout, we let $A$ be a closed subalgebra of $O(\Omega), B$ the smallest stable algebra containing $A$ and $(\triangle B, \mathscr{E}, \mathscr{S})$ the regular triple associated to $A$, with $\pi: \Delta B \rightarrow \Delta B / \mathscr{E}$ the quotient map.

We turn first to our first test problem. It is easy to show that $A$ is local on $\Delta A$; in fact, we establish more: $A$ is not only closed under "local belonging" but also under "local approximation".

THEOREM 4. Let $g$ be a continuous function on $\Delta A$. Assume that for each $x$ in $\Delta A$ there are a neighborhood $U$ of $x$ and a sequence $\left\{f_{n}\right\}$ in $A$ such that $\hat{f}_{n} \rightarrow g$, uniformly on $U$. Then $g$ is the Gelfand transform of an element of $A$.

Proof. Since $\triangle A$ and $\Delta B / \mathscr{E}$ agree as sets, and the topology of $\Delta B / \mathscr{E}$ is finer, $g$ is continuous on $\Delta B / \mathscr{E}$. Notice that if $\hat{f}_{n} \rightarrow g$ uniformly on $U$, then $\hat{f}_{n} \rightarrow g$ uniformly on $\pi \pi^{-1}(U)$. Hence $g$ is holomorphic on each of the sets $\pi(W)$, for $W$ an open, relatively compact subset of $\Delta B$, so $g \in \Gamma(\Delta B / \mathscr{E}, \mathscr{S})$. By the classification theorem, $g$ is the Gelfand transform of an element of $A$, as asserted.

The solution to the second test problem is just as easy.

THEOREM 5. Every complex-valued homomorphism of $A$ is continuous.

Proof. Since the zero homomorphism is obviously continuous, we need only consider a homomorphism $\varphi$ for which $\varphi(1)=1$. By [10, VII, C7], we can find functions $f_{1}, f_{2}, f_{3}$ in $A$ sunch that $\left(\hat{f}_{1}, \hat{f}_{2}, \hat{f}_{3}\right)$ : $\Delta A \rightarrow C^{3}$ is a one-to-one map. There is evidently no loss of generality in assuming that $\varphi\left(f_{i}\right)=0$ for each $i$. If the functions $\hat{f}_{1}, \hat{f}_{2}, \hat{f}_{3}$ had no common zero on $\Delta A$, then by a theorem of Arens [2], the functions 
$\hat{f}_{1}, \hat{f}_{2}, \hat{f}_{3}$ would generate an improper ideal in $A$, contradicting the fact that they lie in the kernel of $\varphi$. On the other hand, the functions $\hat{f}_{1}, \hat{f}_{2}, \hat{f}_{3}$ have at most one common zero (since the map $\left(\hat{f}_{1}, \hat{f}_{2}, \hat{f}_{3}\right)$ is one-to-one). Hence there is a unique point $x$ in $\Delta A$ at which $\hat{f}_{1}, \hat{f}_{2}, \hat{f}_{3}$ all vanish. Now, for an arbitrary $f$ in $A$, a similar argument shows that $\varphi(f)=\hat{f}(x)$, so that $\varphi$ is evaluation at $x$, and is thus continuous.

The solution to the third test problem is considerably more involved. We begin by recalling some facts about regular maps and tangential dimension.

Let $\left(X, \mathcal{O}_{X}\right)$ be a complex-analytic space. We say that a holomorphic map $F: X \rightarrow C^{n}$ is regular if each point of $X$ has a neighborhood which is mapped biholomorphically by $F$ onto a closed subvariety of some open set in $C^{n}$. Note that if $X^{\prime}$ is a local subvariety of $X$, then $F \mid X^{\prime}$ is also regular. Moreover, if $X^{\prime}$ is also relatively compact, then there is a positive number $\varepsilon$ such that if $G: X \rightarrow C^{n}$ is holomorphic and $|F(x)-G(x)|<\varepsilon$ for each $x$ in $X$, then $G \mid X^{\prime}$ is also regular. By the tangential dimension of $X$ at the point $x, \operatorname{dimt}_{x} X$, we mean the least integer $n$ for which there are an open neighborhood $U$ of $x$ and a regular map $H: U \rightarrow C^{n}$. By the tangential dimension of $X$ we mean $\operatorname{dimt} X=\sup \left\{\operatorname{dimt}_{x} X: x \in X\right\}$.

Finally, recall that a holomorphic map $F: X \rightarrow C^{n}$ is almost proper if every connected component of $F^{-1}(K)$ is compact for every compact subset $K$ of $\boldsymbol{C}^{n}$.

We will make use of the following lemma, which was originally pointed out to us by Joseph A. Becker. Since a more general result has been obtained by Tarabay [16], we omit the proof.

LEMMA 6. Let $X$ be a one-dimensional complex-analytic space. If each irreducible branch of $X$ is non-singular, and no more than $k$ of them meet at any one point, then $\operatorname{dimt} X \leqq k$.

We can now present a solution to the third test problem.

THEOREM 7. Let $\Omega$ be an open subset of $C$ with $k$ connected components $(1 \leqq k \leqq \infty)$ and let $A$ be a closed subalgebra of $\mathcal{O}(\Omega)$ which contains the polynomials. If $k=1$ or 2 , then $A$ is generated by a set of $k+1$ functions; if $k \geqq 3$ then $A$ is generated by a set of $k$ functions.

Proof. We deal first with the case $k \geqq 3$, and then indicate the changes necessary to deal with the other case. The proof is basically a chase through the proof in [10] of Narasimhan's imbedding theorem [12]. 
Let $B$ be the smallest stable algebra containing $A$. Choose and fix a sequence $\left\{W_{j}\right\}$ of open, relatively compact, $B$-convex subsets of $\Delta B$ such that $\bar{W}_{j} \subset W_{j+1}$ for each $j$ and $\bigcup W_{j}=\Delta B$. We will ignore the difference in topologies and identify $\Delta B / \mathscr{E}$ with $\Delta A$. Let $X_{j}=$ $\pi\left(W_{j}\right)$, regarded as a complex-analytic space via the pseudo-analytic structure $\mathscr{S}$ associated to $A$. For each $m$, let $A^{m}$ denote the product of $m$ copies of $A$. Set

$$
\begin{aligned}
\mathscr{G} & =\{f \in A: f: \Delta B \rightarrow C \text { is an almost proper map }\}, \\
\mathscr{C} & =\left\{\left(f_{1}, f_{2}\right) \in A^{2}:\left(\widehat{z}, \hat{f}_{1}, \hat{f}_{2}\right): \Delta A \rightarrow C \text { is a one-to-one map }\right\}, \\
\mathscr{K}_{j} & =\left\{\left(f_{1}, \cdots, f_{k-1}\right) \in A^{k-1}:\left(\widehat{z}, \hat{f}_{1}, \cdots, \hat{f}_{k-1}\right) \mid X_{j} \text { is regular }\right\}, \\
\mathscr{K} & =\bigcap_{j=1}^{\infty} \mathscr{K}_{j} .
\end{aligned}
$$

We wish to find a $(k-1)$-tuple $\left(g_{1}, \cdots, g_{k-1}\right)$ in $\mathscr{K}$ - such that $g_{1} \in \mathscr{G}$, and $\left(g_{1}, g_{2}\right) \in \mathscr{K} ; A$ will then be generated by $\left\{z, g_{1}, \cdots, g_{k-1}\right\}$.

In order to do this, we want to see that each of $\mathscr{G}, \mathscr{K}, \mathscr{K}$ is a dense $G_{\delta}$ in the appropriate space. For $\mathscr{G}$, we can apply the argument of [10, VII, C2] as soon as we make a simple observation. Let $K$ be a compact $B$-convex subset of $\Delta B$. Then $\pi(K)$ is a compact $A$-convex subset of $\Delta A$, so $\pi^{-1} \pi(K)$, which is the $A$-convex hull of $K$ in $\Delta B$, is a closed set which is the union of $K$ and a countable set. Hence there is a relatively compact open subset $U$ of $\Delta B$ which contains $K$ and whose boundary misses $\pi^{-1} \pi(K)$. The standard argument now allows us to find an analytic polyhedron $P$ in $\Delta B$, defined by functions in $A$, such that $U \supset P \supset K$. Proceeding exactly as in [10, VII, C2] we can conclude that $\mathscr{G}$ is a dense $G_{\delta}$ in $A$.

To see that $\mathscr{H}$ is a dense $G_{\dot{\delta}}$ in $A^{2}$, we need only apply the remarks following [10, VII, C8].

To show that $\mathscr{K}$ is a dense $G_{\delta}$ in $A^{k-1}$, it suffices (by the Baire Category Theorem) to show that each $\mathscr{K}_{j}$ is a dense open set. Openness of $\mathscr{K}_{j}$ follows immediately from our remarks about regular mappings. To see that $\mathscr{K}_{j}$ is dense, fix $\left(f_{1}, \cdots, f_{k-1}\right)$ in $A^{k-1}$. Let $\varepsilon$ be an arbitrary positive number and let $\ell$ be an arbitrary integer greater than $j+1$. Since $\Omega$ has $k$ connected components, $\Delta B$ has at most $k$ connected components. Since $\pi$ is one-to-one on each connected component of $\Delta B$, we conclude that each irreducible branch of $X_{\ell+1}$ is non-singular and at most $k$ irreducible branches meet at a point. It follows from Lemma 6 that $\operatorname{dimt} X_{c_{+1}} \leqq k$. By [10, VII, C11] we can find holomorphic functions $h_{1}, \cdots, h_{k-1}$ on $X_{\ell+1}$ such that $\left(\widehat{z}, h_{1}, \cdots, h_{k-1}\right): X_{\ell+1} \rightarrow C^{k}$ is regular and $\left\|h_{i}-\hat{f}_{i}\right\|_{\bar{x}}<\varepsilon / 2$ for each $i$. As we showed in the proof of the classification theorem, $\hat{A} \mid X_{\ell+1}$ is dense in $\mathcal{O}\left(X_{t+1}\right)$, so we can choose functions $g_{1}, \cdots, g_{k-1}$ in $A$ such that $\left\|\hat{g}_{i}-h_{i}\right\|_{\vec{X}}<\varepsilon / 2$ and $\left(\widehat{z}, \hat{g}_{1}, \cdots, \widetilde{g}_{k-1}\right): X_{\ell-1} \rightarrow C^{k}$ is regular. Since 
$\iota>j+1$, we conclude that $\left(g_{1}, \cdots, g_{k-1}\right) \in \mathscr{K}_{j}$ and that $\left\|g_{i}-f_{i}\right\|_{\bar{w}_{e}}=$ $\left\|\hat{g}_{i}-\hat{f}_{i}\right\|_{\bar{x}_{\ell}}<\varepsilon$. Since $\measuredangle$ and $\varepsilon$ are arbitrary, we conclude that $\mathscr{K}_{j}$ is dense, so $\mathscr{K}$ is a dense $G_{\delta}$, as desired.

We have now concluded that each of $\mathscr{G}, \mathscr{H}, \mathscr{K}$ is a dense $G_{\dot{\delta}}$; another application of the Baire Category Theorem allows us to conclude that there is an element $\left(g_{1}, \cdots, g_{k-1}\right)$ of $\mathscr{K}$ such that $g_{1} \in \mathscr{G}$ and $\left(g_{1}, g_{2}\right) \in \mathscr{H}$. Let $A_{0}$ be the closed subalgebra of $A$ generated by $\left\{z, g_{1}, \cdots, g_{k-1}\right\}$; we claim that $A_{0}=A$. To see this, we wish to use the classification theorem.

Let $B_{0}$ be the smallest stable algebra containing $A_{0}$; our first task is to see that $B_{0}=B$. Clearly $B_{0} \subset B$, so by Theorem 1 we need only show that the restriction $r: \Delta B \rightarrow \Delta B_{0}$ is one-to-one and onto. It follows from Theorem 1 that $r$ is one-to-one on each connected component; since $\Delta B$ and $\Delta B_{0}$ are manifolds, either $r$ is oneto-one or it identifies uncountably many pairs of points. But since $z, g_{1}, g_{2}$ belong to $A_{0}$ and separate all but countably many pairs of points of $\Delta B$, we conclude that $r$ is in fact one-to-one. At this point it will be convenient to suppose $r$ and simply regard $\Delta B$ as an open subset of $\Delta B_{0}$. Let $C$ be a connected component of $\Delta B$ and let $C_{0}$ be the connected component of $\Delta B_{0}$ which contains $C$. If $C \neq C_{0}$, let $x$ be a boundary point of $C$ in $C_{0}$. Regard $g_{1}$ as a map from $\Delta B_{0}$ into $C$, and let $D$ be the connected component of $g_{1}^{-1}\left\{z:\left|z-g_{1}(x)\right|<1\right\}$ which contains $x$; since $\Delta B_{0}$ is locally connected, $D$ is open. Since $g_{1}: \Delta B \rightarrow C$ is an almost proper map, each connected component of $D \cap C$ has compact closure in $C$. Let $D_{1}$ be any such component; then the boundary of $D_{1}$ (relative to $C_{0}$ ) is contained in $C$. Since $D_{1}$ is closed subset of $D \cap C$, it follows that the boundary of $D_{1}$, relative to $D$, is empty; i.e., $D_{1}$ is closed in $D$. Since $D_{1}$ is open and $D$ is connected, we conclude that $D_{1}=D$, which is absurd, since $D$ contains $x$ and $D_{1}$ does not. We conclude that $C=C_{0}$. Finally, observe that every component of $\Delta B_{0}$ contains a component of $\Omega$ and hence contains a component of $\Delta B$. We conclude that $\Delta B=\Delta B_{0}$, so desired.

It now follows immediately from our construction that the algebras $A$ and $A_{0}$ induce the same equivalence relations and pseudo-analytic structures. By the classification theorem, we conclude that $A_{0}=A$, as desired. This completes the proof in the case $k \geqq 3$.

If $k=2$, the same argument as above applies, although we will still need the function $z$ and two other functions to separate the points of $\triangle A$, so we will obtain a set of three functions which generates $A$. Finally, if $k=1$, the function $z$ and any almost proper function will generate $A$ (see also [19, Corollary 3]).

It is worth noting that the above argument can be easily modified to provide a necessary and sufficient condition that a closed subalgebra 
$A$ (containing the polynomials) of $O(\Omega)$ be finitely generated, where $\Omega$ is an arbitrary open subset of $C$ : the algebra $A$ is finitely generated if and only if $\sup \{\operatorname{dimt} \pi(W)\}<\infty$, where the supremum extends over all open, relatively compact subsets $W$ of $\Delta B$.

We conclude this section by giving one further application of our classification scheme; it is suggested by work of Gamelin [8] and Bjork and de Paepe [7].

THEOREM 8. Let $A$ be a closed subalgebra of $\mathcal{O}(\Omega)$ which contains the polynomials and let $B$ be the smallest stable algebra containing $A$. Then there are closed algebras $A_{0}, A_{1}, \cdots$ such that:

(i ) $B=A_{0} \supset A_{1} \supset A_{2} \supset \cdots \supset A$;

(ii) $A=\bigcap A_{j}$;

(iii) $A_{j+1}$ is of finite co-dimension in $A_{j}$, for each $j \geqq 0$;

(iv) $A_{j+1}$ is a maximal subalgebra of $A_{j}$, for each $j \geqq 0$.

Proof. Choose and fix an increasing sequence $\left\{W_{j}\right\}$ of subsets of $\Delta B$ such that $\Delta B=\bigcup W_{j}$. Set $A_{0}=B$, and for each $j \geqq 1$, let $A_{j}$ be the set of functions $f$ in $B$ for which there is a holomorphic function $\hat{f}$ on $\pi\left(W_{j}\right)$ such that $f\left|W_{j}=\hat{f} \circ \pi\right| \pi\left(W_{j}\right)$. It is easy to see that each $A_{j}$ is a closed subalgebra of $B$ and that $A_{j} \supset A_{j+1}$ for each $j$. The classification scheme guarantees that $A \subset A_{j}$ for each $j$ and that $\bigcap A_{j}=A$, so the algebras $\left\{A_{j}\right\}$ satisfy (i) and (ii). To see that they also satisfy (iii), note that for each $i \geqq 1$, the fact that $W_{i}$ is relatively compact guarantees that $\pi\left(W_{i}\right)$ is a one-dimensional analytic space with only a finite number of singularities. Hence the subalgebra $\left\{g \circ \pi: g \in \mathcal{O}\left(\pi\left(W_{i}\right)\right)\right\}$ of $\mathcal{O}\left(W_{i}\right)$ is of finite co-dimension. It follows immediately that $A_{i}$ is of finite co-dimension in $B$. Writing $i=j+1$ for $j \geqq 0$, it follows a fortiori that $A_{j+1}$ is of finite codimension in $A_{j}$, which yields (iii). Finally, if for some indices $j$, $A_{j+1}$ is not a maximal subalgebra of $A_{j}$, we may remedy this defect by simply interpolating a finite number of algebras between $A_{j+1}$ and $A_{j}$, thereby achieving (iv).

4. Algebras on Riemann surfaces. It is natural to seek a classification theorem for uniform algebras on arbitrary (open) Riemann surfaces which parallels the one given here for algebras on plane domains. Although we believe that no really satisfactory classification scheme exists in this generality, it is actually possible to say quite a lot.

Throughout this section, we let $R$ be an open (not necessarily connected) Riemann surface and $A$ a closed subalgebra of $\mathscr{O}(R)$ which contains the constants and separates the points of $R$. We let $\delta: R \rightarrow \Delta A$ be the evaluation map. Our first task is to find a reason- 
able substitute for Theorems 1 and 2. Fortunately, such a result has been established by Bishop [5] and Royden [14]; it may by formulated as follows.

THEOREM 9. There is an open Riemann surface $\mathscr{S}$ containing $R$ and a continuous map $\tilde{\delta}: \mathscr{S} \rightarrow \Delta A$ with the following properties:

(i) every connected component of $\mathscr{S} \backslash R$ is compact;

(ii) every $f \in A$ has a unique extension $\hat{f} \in \mathcal{O}(\mathscr{S})$;

(iii) $\tilde{\delta}$ is onto and $\tilde{\delta} \mid R=\delta$;

(iv) if $W$ is a relatively compact, open subset of $\mathscr{S}$ and $\mathscr{S} \backslash W$ has no compact components, then $\widetilde{\delta}(W)$ is an A-convex subset of $\triangle A$;

(v) $\mathscr{E}_{A}=\{(x, y) \in \mathscr{S} \times \mathscr{S}: \tilde{\delta}(x)=\tilde{\delta}(y)\}$ is an admissible equivalence relation.

The difference between the above result and Theorems 1 and 2 lies in the extent of our knowledge of the Riemann surface $\mathscr{S}$. In the previous context, we were able to obtain very precise information about $\mathscr{S}$ and drastically limit the possibilities. In the current context, the possibilities for $\mathscr{S}$ are limited only by condition (i). To illustrate just how weak a limitation this is, we present a result which has the status of a "folk theorem"; it is certainly "wellknown" - at least to many people-but does not seem to be formulated in the literature.

Proposition 10. Let $\mathscr{S}$ be a connected, open Riemann surface. Then there is an open, connected subset $\Omega$ of $\mathscr{S}$ such that:

(i) each component of $\mathscr{S} \backslash \Omega$ is compact;

(ii) the restriction map $\mathscr{O}(\mathscr{S}) \rightarrow \mathscr{O}(\Omega)$ is one-to-one with closed range, so that $\mathscr{O}(\mathscr{S})$ is isomorphic to $\mathscr{O}(\mathscr{S}) \mid \Omega$;

(iii) $\Omega$ is biholomorphically equivalent to a plane domain.

Proof. The structure theory of open Riemann surfaces (see [1]) guarantees that we can choose a countable discrete family $\left\{\Gamma_{n}\right\}$ of disjoint simple closed curves in $\mathscr{S}$ which represent a free basis for the homology group $H_{1}(\mathscr{S}, \boldsymbol{Z})$. Let $J$ be the set of indices $n$ for which $\Gamma_{n}$ does not separate $\mathscr{S}$, and set $E=\bigcup_{n \in J} \Gamma_{n}$. Since $\left\{\Gamma_{n}\right\}$ is a discrete family of curves, $E$ is a closed set, $\Omega=\mathscr{S} \backslash E$ is open and every connected component of $\mathscr{S} \backslash \Omega$ is compact. Hence the restriction $\mathscr{O}(\mathscr{S}) \rightarrow \mathscr{O}(\Omega)$ is one-to-one with closed range. Since $\left\{\Gamma_{n}\right\}$ forms a free basis for $H_{1}(\mathscr{S}, Z)$, and we have removed precisely the curves which do not separate $\mathscr{S}, \Omega$ is connected and every simple closed curve in $\Omega$ separates $\Omega$. Thus $\Omega$ is biholomorphically equivalent to a plane domain, as desired.

Thus, for subalgebras of $\mathscr{O}(R)$ we will in general be able to say 
very little about the Riemann surface $\mathscr{S}$, and it is for this reason that we have formulated our main results for algebras (containing the polynomials) on plane domains. However, if we are willing to content ourselves with a poor description of the Riemann surface $\mathscr{S}$, the remainder of the classification scheme is easily obtained. Since the construction of $\S 2$ goes through virtually without change, we omit the details.

THEOREM 11. Let $R$ be an open Riemann surface. Then there is a bijection between the set of closed subalgebras of $\mathcal{O}(R)$ which contain the constants and separate the points of $R$, and the triples $(\mathscr{S}, \mathscr{E}, \mathscr{S})$, where $\mathscr{S}$ is an open Riemann surface containing $R$ and having the property that each component of $S \backslash R$ is compact, $\mathscr{E}$ is an admissible equivalence relation on $\mathscr{S}$ and $\mathscr{S}$ is a pseudo-analytic structure on $\mathscr{S} / \mathscr{E}$.

Although we regard this classification theorem as unsatisfactory (because we cannot obtain a sufficiently precise description of $\mathscr{S}$ ), it is actually good enough for a number of applications. In fact, the proofs of Theorems 4 and 5 go through verbation. However, Theorem 7, which is probably the most striking application, no longer holds in the more general context, as the following simple example demonstrates. Let $\boldsymbol{N}$ denote the set of positive integers. Let $A_{0}$ denote the set of entire functions which vanish to order at least $n$ at the point $n$ (for each $n$ ), and $A$ the closed subalgebra of $\mathscr{O}(\boldsymbol{C})$ spanned by $A_{0}$ and the constants. Then $A \mid(C \backslash N)$ is a point-separating subalgebra of $\mathcal{O}(\boldsymbol{C} \backslash N)$, and, as is easily seen, is not finitely-generated.

\section{REFERENCES}

1. L. Ahlfors and L. Sario, Riemann Surfaces, Princeton Univ. Press, Princeton, N.J. (1960).

2. R. Arens, Dense inverse-limit rings, Mich. Math. J., 5 (1958), 169-182.

3. E. Bishop, Subalgebras of functions on a Riemann surface, Pacific J. Math., 8 (1958), 29-52.

4. —, Partially analytic spaces, Amer. J. Math., 83 (1961), 669-692.

5. - Analyticity in certain Banach algebras, Trans. Amer. Math. Soc., 102 (1962), 507-544.

6. - Holomorphic completion, analytic continuation, and the interpolation of semi-norms, Ann. Math., 78 (1963), 468-500.

7. J.-E. Bjork and P. de Paepe, Maximal subalgebras, Papers from the Summer Gathering on Function Algebras at Aarhus, July 1969, Various Publications Series, no. 9 (1969).

8. T. Gamelin, Embedding Riemann surfaces in maximal ideal spaces, J. Functional Ananlysis, 2 (1968), 123-146.

9. - Polynomial approximation on thin sets, Symposium on Several Complex Variables, Park City, Utah, 1970, Lecture Notes in Math., vol. 184, Springer-Verlag, New York (1971), 50-78. 
10. R. Gunning and H. Rossi, Analytic Functions of Several Complex Variables, Prentice-Hall, Englewood Cliffs, N.J. (1965).

11. I. Kaplansky, Infinite Abelian Groups, Univ. of Mich. Press, Ann Arbor, Math. (1954).

12. R. Narasimhan, Imbedding of holomorphically complete complex spaces, Amer. J. Math., 82 (1960), 917-934.

13. - Introduction to the Theory of Analytic Spaces, Lecture Notes in Math., vol. 25, Springer-Verlag, New York (1966).

14. H. Royden, Algebras of bounded analytic functions on Riemann surfaces, Acta. Math., 114 (1965), 113-141.

15. E. L. Stout, The Theory of Uniform Algebras, Bogden and Quigley, Tarrytown-onHudson, New York (1971).

16. A. Tarabay. Dissertation, University of Utah (1979).

17. J. Wermer, Function rings and Riemann surfaces, Ann. Math. (2), 67 (1958), 45-71.

18. - Rings of analytic functions, Ann. Math. (2), 67 (1958), 497-516.

19. W. Zame, Algebras of analytic functions in the plane, Pacific J. Math., 42 (1972), 811-819.

20. - Uniform algberas on plane domains, several complex variables, Proc. Symposia Pure Math., vol. 30, Amer. Math. Soc., Providence, R.I., (1970), 193-196.

Received January 9, 1980 and in revised form October 7, 1980. Supported in part by National Science Foundation Grants PO 37961-001, MCS 79-01786 and MCS 77-18723 A 03.

SUNY

BUfFaLo, NY 14214 



\section{PACIFIC JOURNAL OF MATHEMATICS}

\section{EDITORS}

DONALD BABBITT (Managing Editor)

University of California

Los Angeles, California 90024

\section{Hugo Rossi}

University of Utah

Salt Lake City, UT 84112

C. C. MOore and Arthur AGuS

University of California

Berkeley, CA 94720
J. DugundJI

Department of Mathematics University of Southern California Los Angeles, California 90007

R. FinN and J. Milgram Stanford University Stanford, California 94305

ASSOCIATE EDITORS
R. ARENS
E. F. BECKENBACH
B. H. NeumanN
F. WOLF
K. YOSHIDA

\section{SUPPORTING INSTITUTIONS}

UNIVERSITY OF ARIZONA

UNIVERSITY OF BRITISH COLUMBIA

CALIFORNIA INSTITUTE OF TECHNOLOGY

UNIVERSITY OF CALIFORNIA

MONTANA STATE UNIVERSITY

UNIVERSITY OF NEVADA, RENO

NEW MEXICO STATE UNIVERSITY

OREGON STATE UNIVERSITY
UNIVERSITY OF OREGON

UNIVERSITY OF SOUTHERN CALIFORNIA

STANFORD UNIVERSITY

UNIVERSITY OF HAWAII

UNIVERSITY OF TOKYO

UNIVERSITY OF UTAH

WASHINGTON STATE UNIVERSITY

UNIVERSITY OF WASHINGTON 


\section{Pacific Journal of Mathematics}

Vol. 99, No. $1 \quad$ May, 1982

Mariano Giaquinta, Jindrich Necas, O. John and J. Stará, On the

regularity up to the boundary for second order nonlinear elliptic systems . . 1

Siegfried Graf, Realizing automorphisms of quotients of product $\sigma$-fields . . 19

Alfred Washington Hales and Ernst Gabor Straus, Projective colorings . . . 31

Sandra Hayes, The weak Nullstellensatz for finite-dimensional complex

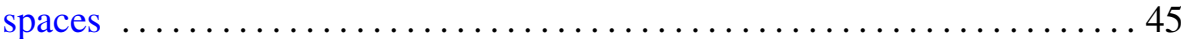

Gerald Norman Hile and Murray Harold Protter, The Cauchy problem

and asymptotic decay for solutions of differential inequalities in Hilbert

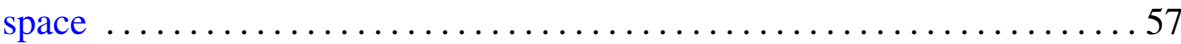

Robert D. Little, Projective space as a branched covering with orientable

branch set ......................................... 89

Jaroslav Mach, On the proximinality of Stone-Weierstrass subspaces . . . . . 997

John C. Morgan, II, On product bases ...................... 105

K. Balakrishna Reddy and P. V. Subrahmanyam, Altman's contractors

and fixed points of multivalued mappings . .................. 127

James Ted Rogers Jr., Decompositions of homogeneous continua . . . . . . . 137

Ahmed Ramzy Sourour, Characterization and order properties of

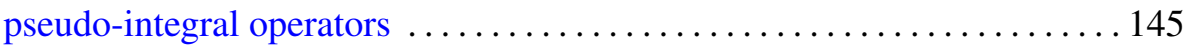

Robert Moffatt Stephenson Jr., Pseudocompact and Stone-Weierstrass

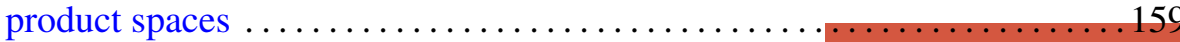

Bruce Stewart Trace, On attaching 3-handles to a 1-connected

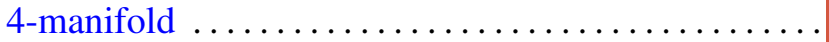

Akihito Uchiyama, The construction of certain BMO functions and the corona problem

Thomas Alva Whitehurst, An application of orthogonal polynomials to random walks ..............................

David J. Winter, Root locologies and idempotents of Lie and nonassociative algebras

William Robin Zame, The classification of uniform algebras on plane domains 